\title{
Genetic variants in VEGF pathway genes in neoadjuvant breast cancer patients receiving bevacizumab: results from the randomized phase 3 GeparQuinto study
}

Alexander Hein ${ }^{* 1}$, Diether Lambrechts* ${ }^{2,3}$, Gunter von Minckwitz ${ }^{4,5}$, Lothar Häberle ${ }^{1,6}$, Holger Eidtmann ${ }^{7}$, Hans Tesch ${ }^{8}$, Michael Untch ${ }^{9}$, Jörn Hilfrich ${ }^{10}$, Christian Schem $^{7}$, Mahdi Rezai ${ }^{11}$, Bernd Gerber ${ }^{12}$, Serban Dan Costa ${ }^{13}$, Jens-Uwe Blohmer ${ }^{14}$, Kathrin Schwedler ${ }^{5,15}$, Kornelia Kittel ${ }^{16}$, Tanja Fehm ${ }^{17,18}$, Georg Kunz ${ }^{19}$, Matthias W. Beckmann $^{1}$, Arif B. Ekici ${ }^{20}$, Claus Hanusch ${ }^{21}$, Jens Huober ${ }^{22}$, Cornelia Liedtke ${ }^{23}$, Christine Mau', Matthieu Moisse ${ }^{2}$, Volkmar Müller ${ }^{24}$, Valentina Nekljudova ${ }^{4}$, Gilian Peuteman ${ }^{2}$, Brigitte Rack ${ }^{25}$, Matthias Rübner ${ }^{1}$, Thomas Van Brussel ${ }^{2}$, Liewei Wang ${ }^{26}$, Richard M. Weinshilboum ${ }^{26}$, Sibylle Loibl ${ }^{4,5}$, Peter A. Fasching ${ }^{1,27}$

* These authors contributed equally to the study.

1. Department of Gynecology and Obstetrics, University Breast Center for Franconia, Erlangen University Hospital, Friedrich-Alexander University of Erlangen-Nuremberg, Erlangen-EMN Comprehensive Cancer Center, Erlangen, Germany

2. Vesalius Research Center, VIB, Leuven, Belgium

3. Laboratory of Translational Genetics, Department of Oncology, University of Leuven, Leuven, Belgium

4. GBG Forschungs $\mathrm{GmbH}$, Neu-Isenburg, Germany

5. Department of Gynecology and Obstetrics, Frankfurt University Hospital, Goethe University of Frankfurt, Frankfurt am Main, Germany

6. Biostatistics Unit, Department of Gynecology and Obstetrics, Erlangen University Hospital, Germany

7. Department of Obstetrics and Gynecology, Christian-Albrechts-University, Kiel, Germany

8. Onkologie Bethanien, Frankfurt, Germany

9. Helios-Klinikum, Berlin-Buch, Germany

10. Eilenriede-Klinik, Hannover, Germany

11. Breast Center of Düsseldorf, Luisenkrankenhaus, Düsseldorf, Germany

12. Department of Gynecology, University of Rostock, Rostock, Germany

13. Department of Obstetrics and Gynecology, Magdeburg University Hospital, Magdeburg, Germany

14. St. Getrauden Breast Center, St. Gertrauden Krankenhaus, Germany

15. Neue Frauenklinik, Lucerne Cantonal Hospital, Lucerne, Switzerland 
16. Praxisklinik, Krebsheilkunde für Frauen, Brustzentrum, Berlin

17. Department of Gynecology, University of Tübingen, Tübingen, Germany

18. Department of Gynecology and Obstetrics, Düsseldorf University Hospital, Heinrich Heine University, Düsseldorf, Germany

19. St. Johannes Hospital, Dortmund, Germany

20. Institute of Human Genetics, Erlangen University Hospital, Friedrich Alexander University of ErlangenNuremberg, Erlangen, Germany

21. Department of Gynecology, Klinikum zum Roten Kreuz, Munich, Germany

22. Department of Gynecology, University of UIm, Ulm, Germany

23. Department of Gynecology and Obstetrics, Lübeck University Hospital, Lübeck, Germany

24. Department of Gynecology and Obstetrics, Hamburg University Hospital, Hamburg, Germany

25. Department of Gynecology and Obstetrics, Ludwig Maximilian University, Munich, Germany

26. Department of Molecular Pharmacology and Experimental Therapeutics, Division of Clinical Pharmacology, Mayo Clinic College of Medicine, Mayo Medical School-Mayo Foundation, Rochester, Minnesota, USA

27. Department of Medicine, Division of Hematology/Oncology, University of California at Los Angeles, David Geffen School of Medicine, Los Angeles, California, USA

\section{Corresponding author:}

Peter A. Fasching, MD; Department of Gynecology and Obstetrics, Erlangen University Hospital, Friedrich Alexander University of ErlangenNuremberg/Comprehensive Cancer Center, Universitaetsstrasse 21-23, 91054 Erlangen, Germany; E-mail: peter.fasching@uk-erlangen.de

\section{Running title:}

VEGF SNPs and bevacizumab response in the GeparQuinto trial

\section{Novelty \& Impact Statements:}

In this pre-planned biomarker study of the neoadjuvant GeparQuinto trial, germline genetic variants in VEGF pathway genes were associated with bevacizumab treatment outcome, as assessed by pathological complete response. In particular, four variants in VEGFA and one variant in FLT1 were associated with treatment outcome. The effect of some variants seemed more pronounced in patients with triple-negative tumors. 


\section{Abstract}

Studies assessing the effect of bevacizumab (BEV) on breast cancer outcome have shown different effects on progression-free and overall survival, suggesting that a subgroup of patients may benefit from this treatment. Unfortunately, no biomarkers exist to identify these patients. Here, we investigate whether single nucleotide polymorphisms (SNPs) in VEGF pathway genes correlate with pathological complete response ( $\mathrm{pCR}$ ) in the neoadjuvant GeparQuinto trial.

HER2-negative patients were randomized into treatment arms receiving either BEV combined with standard chemotherapy or chemotherapy alone. In a pre-planned biomarker study, DNA was collected from 729 and 724 patients, respectively from both treatment arms, and genotyped for 125 SNPs. Logistic regression assessed interaction between individual SNPs and both treatment arms to predict pCR.

Five SNPs may be associated with a better response to BEV, but none of them remained significant after correction for multiple testing. The two SNPs most strongly associated, rs833058 and rs699947, were located upstream of the VEGFA promoter. Odds ratios for the homozygous common, heterozygous and homozygous rare rs833058 genotypes were $2.36(95 \% \mathrm{Cl}, 1.49-3.75), 1.20(95 \% \mathrm{Cl}, 0.88-1.64)$ and $0.61(95 \% \mathrm{Cl}, 0.34-1.12)$. Notably, some SNPs in VEGFA exhibited a more pronounced effect in the triple-negative subgroup.

Several SNPs in VEGFA may be associated with improved $\mathrm{pCR}$ when receiving BEV in the neoadjuvant setting. Although none of the observed effects survived correction for multiple testing, our observations are consistent with previous studies on BEV efficacy in breast cancer. Further research is warranted to clarify the predictive value of these markers.

Key words: breast cancer, VEGF, anti-angiogenesis, bevacizumab, genetic variant, prognosis, biomarker, SNP 


\section{Introduction}

Bevacizumab (BEV), a monoclonal antibody vascular endothelial growth factor-A (VEGFA), was the first anti-angiogenic drug to be approved for the treatment of breast cancer. Although it slows the progression of metastatic breast cancer when combined with chemotherapy, ${ }^{1-4}$ the Food and Drug Administration withdrew approval for this indication in 2011 due to (i) a lack of evidence for a consistent overall survival (OS) benefit, (ii) the frequent occurrence of BEV-related toxicities, and (iii) the fact that there are no validated biomarkers to predict which patients really benefit from the therapy. ${ }^{5}$ Although the therapeutic effect of BEV indeed appears to be modest in the metastatic breast cancer setting, the European Medicines Agency maintained its approval status. Similarly, recent neoadjuvant studies have shown a modest but significant increase in the pathological complete response (pCR) in HER2-negative breast cancer patients. ${ }^{6,7}$ In adjuvant studies, however, no benefit with regard to the disease-free survival has been demonstrated for patients with triple-negative (TN) or HER2-positive breast cancer (BC). ${ }^{8,9}$

Markers capable of predicting the response to anti-angiogenic treatment or the occurrence of side effects are thus urgently needed, as this might improve patient selection for these patients. With regard to breast cancer subtypes, the GeparQuinto trial has shown that patients with triple-negative breast cancer (TNBC) appear to benefit more from BEV treatment than non-TNBC patients. ${ }^{6}$ However, this effect was not seen in the NSABP-B40 study. ${ }^{7}$ Several other biomarkers predictive of BEV treatment were identified in retrospective studies, including high levels of tumor-associated VEGF or high circulating levels of plasma VEGF. ${ }^{8,10-12}$ In another study, gene amplifications involving VEGFA were associated with a poor outcome specifically in the subgroups of triple-negative and HER2-positive breast cancers. ${ }^{13}$

In this study, we hypothesize that germline genetic variants underlie the response to the anti-angiogenic effects of BEV. Angiogenesis is a process that affects tumor microenvironment and by affecting vessel regression and permeability only indirectly affects the tumor itself. Since endothelial cells, unlike tumor cells, are not affected by somatic alterations, germline genetic variants could indeed explain why some patients respond and others do not respond to anti-angiogenic therapy. Various potential predictive genetic markers have been identified in clinical trials evaluating BEV in several tumor types, ${ }^{13-19}$ but these analyses - generally using single-trial datasets - are limited by the number of available samples, heterogeneity in the selection of single nucleotide polymorphisms (SNPs), and a lack of understanding of the functional consequences of these genetic markers. An analysis of two 
randomized phase III trials with BEV in metastatic pancreatic adenocarcinoma (AViTA) and metastatic renal-cell carcinoma (AVOREN) has shown a significant correlation between rs9582036, a SNP in FLT1, and both PFS and OS in the BEV group only. ${ }^{16}$ Trials in metastatic colorectal cancer have also reported significant associations with SNPs and BEV efficacy, ${ }^{14,} 15$ although these results often lack confirmation. ${ }^{17}$ With respect to breast cancer, a retrospective analysis of the phase 3 E2100 trial involving BEV revealed an association between several VEGF SNPs and either OS (rs699947 and rs1570360) or severe hypertension ( $r$ 2010963). ${ }^{19}$ In another single-arm trial (MO19391) including 137 women, it was confirmed that rs2010963 in VEGFA is related to BEV-associated toxicity, whereas rs3025039 in VEGFA tended to be associated with time-to-progression. A recent meta-analysis of 6 different cancer types, including breast cancer, has also shown that several VEGF SNPs were predictive of PFS and also functionally relevant because they affected VEGFA gene expression, although observed effects were generally quite modest. ${ }^{20}$

The primary aim of this study was to assess whether SNPs in VEGF pathway genes are associated with efficacy of bevacizumab in all patients participating to the neoadjuvant breast cancer study, GeparQuinto, and as a secondary aim whether specific breast cancer subgroups, as defined by hormone receptor status, were more strongly associated to specific genetic variants.

\section{Material and methods}

\section{Study population}

The GeparQuinto phase III trial (NCT00567554) recruited patients with HER2-positive breast cancer for an anti-HER2 therapy-related study aim, ${ }^{21}$ and HER2-negative patients with the aim of testing the efficacy of BEV. ${ }^{6,22}$ In the present study only HER2-negative patients from the GeparQuinto trial were included. The patients were treated with anthracycline and taxane neoadjuvant chemotherapy and were randomly assigned to either simultaneous treatment with bevacizumab or no additional therapy. In total, 1984 patients were randomly assigned in the main study. Germline DNA was available for 1503 of these patients. Some patients were excluded according to the following hierarchical order: patients without final surgery $(n=5)$, patients with $>20 \%$ missing genotype, patient or tumor data $(n=45)$. This resulted in a final sample size of 1453 patients. Of these patients, 729 were treated with bevacizumab and 724 were assigned to the control arm. The pharmacogenetic analysis was a preplanned biomarker substudy. All of the patients provided written informed consent for the pharmacogenetic analysis, and the relevant ethics committees at the participating study sites approved the study protocol. 


\section{Treatment and assessment of clinical data}

The assessment of the clinical data has been described in detail elsewhere. ${ }^{6}$ Briefly, patients included in this study were HER2-negative and scheduled to receive epirubicin and cyclophosphamide on day 1 and every 3 weeks for a total of four cycles, followed by docetaxel as well on day 1 and every 3 weeks for a total of four cycles. Patients were randomly assigned to receive either bevacizumab plus chemotherapy or chemotherapy alone. If there was no sonographical response after four cycles, patients were taken off treatment and continued with a different treatment. ${ }^{23}$ For the present study, however, the patients were analyzed according to the planned therapy intention if they received at least one cycle of chemotherapy or at least one cycle of BEV. All clinical and histopathological data were assessed by the local pathologists, including tumor stage, HER2 status, estrogen receptor/progesterone receptor status, and grading. Pathological reports were assessed and reviewed centrally for the primary study aim. For this analysis, pathological response was defined as pathological stage pTO and pNO after therapy.

\section{DNA extraction and genotyping}

Whole blood samples were collected in citrate-phosphate-dextrose-adenine (CPDA) tubes (Sarstedt AG, Nümbrecht, Germany) from patients who had consented to participate in the biomarker substudy. Germline DNA was extracted using the automated magnetic bead-based chemagic MSM I technique (PerkinElmer chemagen, Baesweiler, Germany) in accordance with the manufacturer's instructions. Genotyping was done at the Vesalius Research Center, Leuven, Belgium using MassARRAY iPLEX Gold (Sequenom, San Diego, California, USA), as reported previously. $^{16}$

\section{Selection of SNPs}

SNPs in the following 15 genes involved in the VEGF-A pathway were selected: VEGFA, the VEGFA homologs (placental growth factor [PGF], VEGFB, VEGFC, and $V E G F D$ [also known as c-fos-induced growth factor or FIGF]), VEGF receptor-1 (VEGFR1 or FLT1), VEGF receptor-2 (VEGFR2 or KDR), VEGF receptor-3 (VEGFR3 or FLT4) and the neuropilin-1 coreceptor (NRP1), regulators of hypoxia (hypoxiainducible factor- $1 \alpha$ [HIF1A], HIF-2 $\alpha$ [EPAS1], factor inhibiting HIF-1A [HIF1AN or $F I H 1]$, von Hippel-Lindau tumor suppressor [VHL]), and the oxygen sensors (prolyl hydroxylase domain-containing proteins 1 [EGLN2], 2 [EGLN1], and 3 [EGLN3]). A detailed description of the way in which SNPs were selected in these genes has been published previously. ${ }^{16}$ Briefly, genomic sequences $5 \mathrm{~kb}$ upstream of the translation start site and downstream of the $3^{\prime}$ polyadenylation site in each gene were used to select SNPs from the HapMap database (phase 2 public release, no. 22). Common SNPs with a minor allele frequency $\geq 0.1$ and pairwise correlation coefficients 
$\left(r^{2}\right) \leq 0.8$ were selected using the SNP Tagger approach in the Haploview software package. ${ }^{24,25} \mathrm{~A}$ total of 170 tagging SNPs in VEGF pathway genes, as well as 16 SNPs previously associated with bevacizumab efficacy or with circulating VEGF levels, were selected. Of these, 170 were successfully designed and genotyped (Supplementary Table 1). Quality control further removed 30 SNPs with a call rate of $<95 \%$. After exclusion of SNPs with a minor allele frequency $<0.1$ in the study, a final set of 125 SNPs was considered for statistical analysis (Supplementary Table 1).

\section{Statistical analyses}

Logistic regression models were used as described below to investigate the association between the treatment arm and several SNPs relative to pCR. Patients with missing PCR information were excluded. Missing information about patient and tumor characteristics was imputed using single "best guesses" (median value of continuous predictors, the most common value of categorical or ordinal categorical predictors) based on non-missing data across all subjects. Missing genotype values were not imputed, but remained missing. Continuous predictors were used as natural cubic spline functions to describe nonlinear effects as recently described. ${ }^{26}$

For each SNP, a logistic regression model was fitted with the outcome pCR and the predictors SNP (ordinal; 0, 1, 2 minor alleles), treatment arm (bevacizumab versus standard treatment), SNP by treatment arm interaction, as well as the established predictors age (continuous), body mass index (continuous), tumor stage (ordinal), lymph-node status (categorical; pNO versus pN+), grading (ordinal; G1, G2, G3), histology (categorical; ductal, lobular, others), and hormone receptor status (categorical; triple-negative versus not triple-negative). The SNP by treatment arm interaction was included to examine whether the differences between the treatment arms depended on genetic variants. The interaction $P$ values were calculated and then corrected using the Bonferroni-Holm method. Genotype-specific odds ratios (ORs) for the treatment arm, adjusted for the established predictors from above, were estimated for ten SNPs with the smallest uncorrected $P$ values using the interaction models.

The association between hormone receptor, genotypes, and treatment arm with regard to PCR was the secondary study aim. Therefore, for each of the ten SNPs with small interaction $P$ values from above, the interaction model was extended by including the three-way interaction SNP by treatment arm by hormone receptor and the corresponding two-way interactions. The two models were compared using the Akaike information criterion (AIC). A better AIC value for the latter model suggests that the association of treatment and genotype differs between patients with triplenegative tumors and patients without triple-negative tumors. In this case, hormone 
receptor and genotype-specific ORs for the treatment arm were estimated using the extended regression model.

The goodness of fit of the logistic regression models was tested using the Hosmer-Lemeshow $\chi^{2}$ test, where a large $P$ value indicates good calibration.

All of the tests were two-sided, and a $P$ value of $<0.05$ was regarded as statistically significant. Calculations were carried out using the R system for statistical computing (version 3.0.1; R Development Core Team, Vienna, Austria, 2013).

\section{Results}

\section{Patient characteristics}

A total of 1453 patients were included in the analysis. Complete data for patient and tumor characteristics were available for $95.7 \%$ of the patients. Patients and tumor characteristics are shown in Table 1. pCR was correlated with all previously described predictors in the expected direction. Patients with a higher tumor stage were less likely to achieve a $\mathrm{pCR}$, and patients with a negative hormone receptor status or a higher grading were more likely to achieve a pCR (Table 1). The patient characteristics were similar to those in the main study. There were no differences in the $\mathrm{pCR}$ rate between patients who took part in this study and those who did not (data not shown).

\section{Genotype results}

Genotyping results, SNP location, and $P$ values for the Hardy-Weinberg equilibrium analysis are presented in Supplementary Table 1.

The association between treatment and genotype with regard to $\mathrm{PCR}$ was analyzed using logistic regression models as described above. Ten SNPs with the smallest $P$ values for SNP by treatment interaction are shown in Table 2 . None of the SNPS were significant after correction for multiple testing. In other words, it was not shown that a genotype influenced the difference between the treatment arms.

Five of these 10 SNPs are located on chromosome 6 around VEGFA, and the top hit is located upstream from the promoter of VEGFA (Fig. 1). Three of the SNPs were located on chromosome 2 around EPAS1. Two further SNPs were related to FLT1 and EGLN3.

Patients with homozygous common genotypes in VEGFA or EPAS1 appeared to benefit from BEV treatment, whereas patients with at least one minor allele did not (Table 2). For example, in relation to rs833058 (VEGFA), patients with the common 
genotype had a greater benefit from BEV treatment, with an OR of 2.36 (95\% Cl, 1.49 to 3.75), whereas patients with a heterozygous or homozygous rare genotype did not benefit (OR for heterozygous: $1.21 ; 95 \% \mathrm{Cl}, 0.89$ to 1.65; OR for homozygous rare: $0.62,95 \% \mathrm{Cl}, 0.34$ to 1.13 ).

Next, the predictive association between treatment and genotype in these SNPs was assessed in the subgroups of hormone receptor-positive and hormone receptornegative patients. Since this study was performed on HER2-negative patients, all hormone receptor-negative patients represent TNBC patients. The treatment efficacy might depend on hormone receptor status in five out of 10 SNPs with the smallest interaction $P$ values, with a greater effect in the group of TNBC patients (Table 3). Patients with a homozygous common genotype and triple-negative tumors appeared to benefit from BEV treatment, whereas the other patients did not. For example, with regard to SNP rs1374749, the effect of the common genotype on the drug response was much greater in triple-negative patients (OR 3.20; $95 \% \mathrm{Cl}, 1.68$ to 6.07) than in patients with hormone receptor-positive, HER2-negative tumors (OR $0.91 ; 95 \% \mathrm{Cl}, 0.45$ to 1.87$)$.

All of the logistic regression models were well calibrated. The $P$ values in the Hosmer-Lemeshow tests ranged from 0.13 to 0.91 , and the median value was 0.49 .

\section{Discussion}

In this pre-planned biomarker substudy of the neoadjuvant GeparQuinto (GBG44) trial, germline genetic variants in VEGF pathway genes were associated with bevacizumab treatment response as assessed by pCR. Remarkably, out of top 10 SNPs with the smallest $P$ values, 5 SNPs were located in VEGFA. None of these remained statistically significant after adjustment for multiple testing. The effect of some SNPs in VEGF-A appeared to be stronger in patients with triple-negative breast tumors.

A biomarker that is able to predict the efficacy of BEV is urgently needed, particularly for patients with breast cancer, as not all clinical trials have shown clear superiority for the drug. Several trials have assessed the predictive value of circulating VEGF-A levels in serum. Although high VEGF-A serum levels were consistently associated with poor prognosis, they failed as a predictive marker for BEV treatment in a metaanalysis including colorectal, lung, breast and renal cancer patients. ${ }^{27}$ In addition, high expression levels of a VEGF-A splice variant in tumor tissue were associated with more favorable PFS and OS in metastatic breast cancer in a single BEV-treated group. ${ }^{28}$ Also levels of the short isoform VEGF-A, which are currently being prospectively validated in a phase III metastatic breast cancer trial (MERiDiAN), look promising as a predictive biomarker for BEV. ${ }^{29}$ However, despite these concerted 
efforts using a wide range of biological sample types, a validated marker predictive of treatment outcome for BEV has so far not been identified.

In the present study, homozygous carriers of the rare rs833058 variant, which correspond to the AA genotype, showed the strongest association with benefit from BEV treatment. Additional predictive SNPs in VEGF-A were intronic (rs3025030), located in the promoter region (rs699947) or 3'UTR region (rs3025039) of VEGF-A. The rs833058 SNP is in linkage $\left(r^{2}=0.51\right)$ with $r s 699947$ and other SNPs in VEGF-A. Interestingly, AA genotype carriers of both rs833058 and rs699947 are associated with an upstream 18 -nucleotide insertion, ${ }^{30}$ which has previously been linked with low VEGF expression levels both in vitro and in vivo. ${ }^{31,32}$ In addition, expression quantitative trait locus (eQTL) analyses from publicly available data sources show an effect of rs699947 on VEGF-A expression in Caucasians ( $r$ o $=0.263 ; P=0.0060$ ) ${ }^{33}$, thus confirming that this variant is linked to VEGF-A expression. Interestingly, the AA genotype of rs699947 was also associated with better OS in metastatic breast cancer patients when treated with a combination of BEV and paclitaxel, but not with paclitaxel alone (E2100 study). ${ }^{19}$ The same genotype was associated with a better response to BEV in the present GeparQuinto study (OR 2.24; 95\% Cl: 1.31-3.84).

A recent comprehensive study analyzed 195 SNPs in six randomized phase III trials testing the addition of BEV to standard chemotherapy in colorectal, pancreatic, lung, renal, breast, and gastric cancer, involving a total of 716 BEV treated patients and 686 patients receiving placebo. ${ }^{20}$ In this analysis, genetic variants in VEGF-C, EPAS1, IL8RA, but also VEGF-A were predictors of treatment outcome after BEV treatment. ${ }^{20}$ Out of the 195 SNPs analyzed in this meta-analysis, we analyzed 110 SNPs in the GeparQuinto study, which included 729 BEV treated patients and 724 control patients. Our study thus has a comparable sample size, but has the advantage that it only consists of one cancer type evaluated for a uniform endpoint in a single randomized study. As such, GeparQuinto is the first study, in which it is possible to compare a comprehensive set of SNPs for a large set of patients. Intriguingly, when our results are compared with those obtained in the meta-analysis, ${ }^{20}$ the same VEGF-A SNPs identified in GeparQuinto were also significant in the meta-analysis. In particular, AA carriers of rs 833058 were also associated with prolonged PFS in the meta-analysis, whereas both rs3025030 and rs3025039 were significant at $P<0.05$ in the meta-analysis. Moreover, when focusing on the breast cancer trial included in this meta-analysis, i.e., the AVADO phase 3 trial for metastatic breast cancer, both rs699947 and rs3025039 were significant $(P<0.05)$ in a treatment by interaction analysis. $^{20}$

Interestingly, the SNPS in the 3' UTR region of VEGF-A, i.e., rs3025030 and rs3025039, also showed a differential effect in the molecular subgroup analysis, with a greater predictive effect in triple-negative patients. The VEGF-A SNPs close to the 
promoter region did not show this effect. Bearing in mind that none of the interaction $P$ values were significant after adjustment for multiple testing and that the effect of these SNPs on VEGF expression in unknown, this result suggests that the effects through which the SNPs in the promoter region and those in the 3' UTR region are mediated may differ from each other.

Finally, we also observed an association between rs7995976 in FLT1 and pCR after BEV. Interestingly, increased SFLT1 levels have previously been linked with poor outcome after anti-angiogenic therapy in several studies, ${ }^{34,} 35$ whereas a significant correlation between another genetic variant in FLT1, i.e., rs9582036, and outcome after BEV was observed in pancreatic and renal cell carcinoma patients. Since sFLT1 is an endogenous anti-angiogenic factor, tumors exposed to high levels of sFLT1 are indeed not expected to benefit from any additional exposure to anti-angiogenic molecules, such as BEV. Since the functional effect of rs7995976 on FLT1 expression is unknown, it thus remains an outstanding question whether this variant should be considered a true-positive candidate biomarker for BEV treatment. In this context multi-tyrosinkinase inhibitors which target FLT1 might be of interest when assessing BEV response. There are already several clinical trials assessing the addition of sunitinib or sorafenib as a monotherapy after a combination of BEV with standard chemotherapy. ${ }^{36,37}$ Unfortunately, these studies were prematurely closed because of an unfavorable toxicity profile. Interestingly, studies have shown that EPAS1 (HIF2 $\alpha$ ) regulates soluble VEGF receptor-1 (sFLT1) production from macrophages, which contributes to the inhibition of VEGFA during angiogenesis in mouse models. ${ }^{38}$ This mechanism might also explain why we observed an increased response to BEV treatment in homozygous carriers of the common (wild-type) alleles for 3 EPAS1 SNPs.

Overall this study has several strengths and limitations. Firstly, although it is one of the largest single-study analyses from a prospectively randomized bevacizumab trial, the sample size of approximately 600 patients in each treatment arm and a total of 261 patients with $\mathrm{PCR}$ is still too small to identify genetic associations of modest effect size while correcting for multiple testing. As a result, none of the SNPs was statistically significant after adjustment for multiple testing. However, since several other studies involving breast cancer patients treated with BEV observed similar association signals, our results support the view that response to BEV is a complex genetic phenotype that requires additional studies to be carried out in this area, in order not to miss a signal that may have been overseen due to limited power.

In conclusion, we failed to observe a strong association between germline genetic variants in VEGF pathway genes and BEV treatment outcome. However, some SNPS in VEGF-A, which have been shown in other studies to correlate with overall survival and have been shown to be associated with reduced VEGF-A expression, were 
predictive for BEV treatment. Additional studies are therefore warranted to explore the effects of these variants on bevacizumab treatment outcome.

\section{Acknowledgments}

This study was supported by the ELAN fund of the University of Erlangen. The conduct of the main study was funded by Sanofi-Aventis and Roche, Germany. The authors are grateful to Sonja Oeser and Silke Landrith for management of samples and biomaterials. We are grateful to Michael Robertson for professional medical editing services.

\section{Conflicts of interest}

Alexander Hein has received honoraria from F. Hoffmann-La Roche for speaker engagement. Diether Lambrechts has received research funding from F. Hoffmann La Roche related to research into biomarkers for bevacizumab and is mentioned as inventor on patents describing some of these variants as potential biomarker for treatment outcome. Gunter von Minckwitz has received research funding from F. Hoffmann La Roche. Hans Tesch has received honoraria from F. Hoffmann-La Roche, GSK and Novartis. Claus Hanusch is member of speakers' bureau, Advisory Board Member and is conducting trials with financial support from F. Hoffmann-La Roche, Pfizer, Boehringer-Ingelheim, Amgen and Novartis. Jens Huober is Advisory Board Member for F. Hoffmann-La Roche and Novartis. Volkmar Müller has received honoraria from F. Hoffmann-La Roche for speaker engagement. Brigitte Rack has received honoraria from F. Hoffmann-La Roche. Peter A. Fasching has received honoraria from F. Hoffmann-La Roche, Amgen, Pfizer, Novartis and Genomic Health for speaker engagement. PAF is Advisory Board Member for F. Hoffmann-La Roche, Pfizer and Novartis and has received Research Funding from Amgen and Novartis. The other authors have no conflicts of interest to disclose regarding the publication of this paper. 


\section{References}

1. Robert NJ, Dieras V, Glaspy J, Brufsky AM, Bondarenko I, Lipatov ON, Perez EA, Yardley DA, Chan SY, Zhou X, Phan SC, O'Shaughnessy J. RIBBON-1: randomized, double-blind, placebo-controlled, phase III trial of chemotherapy with or without bevacizumab for first-line treatment of human epidermal growth factor receptor 2-negative, locally recurrent or metastatic breast cancer. Journal of clinical oncology : official journal of the American Society of Clinical Oncology 2011;29: 1252-60.

2. Brufsky AM, Hurvitz S, Perez E, Swamy R, Valero V, O'Neill V, Rugo HS. RIBBON-2: a randomized, double-blind, placebo-controlled, phase III trial evaluating the efficacy and safety of bevacizumab in combination with chemotherapy for second-line treatment of human epidermal growth factor receptor 2-negative metastatic breast cancer. Journal of clinical oncology : official journal of the American Society of Clinical Oncology 2011;29: 4286-93.

3. Miles DW, Chan A, Dirix LY, Cortes J, Pivot X, Tomczak P, Delozier T, Sohn JH, Provencher L, Puglisi F, Harbeck N, Steger GG, et al. Phase III study of bevacizumab plus docetaxel compared with placebo plus docetaxel for the first-line treatment of human epidermal growth factor receptor 2negative metastatic breast cancer. Journal of clinical oncology : official journal of the American Society of Clinical Oncology 2010;28: 3239-47.

4. Miller K, Wang M, Gralow J, Dickler M, Cobleigh M, Perez EA, Shenkier T, Cella D, Davidson NE. Paclitaxel plus bevacizumab versus paclitaxel alone for metastatic breast cancer. The New England journal of medicine 2007;357: 2666-76.

5. Administration DoHaHSFaD. Proposal to Withdraw Approval for the Breast Cancer Indication for Avastin (Bevacizumab); FDA-2010-N-0621, vol. 2014: Department of Health and Human Services Food and Drug Administration, 2011.

6. von Minckwitz G, Eidtmann H, Rezai M, Fasching PA, Tesch $\mathrm{H}$, Eggemann H, Schrader I, Kittel K, Hanusch C, Kreienberg R, Solbach C, Gerber B, et al. Neoadjuvant chemotherapy and bevacizumab for HER2negative breast cancer. The New England journal of medicine 2012;366: 299309.

7. Bear HD, Tang G, Rastogi P, Geyer CE, Jr., Robidoux A, Atkins JN, Baez-Diaz L, Brufsky AM, Mehta RS, Fehrenbacher L, Young JA, Senecal FM, et al. Bevacizumab added to neoadjuvant chemotherapy for breast cancer. The New England journal of medicine 2012;366: 310-20.

8. Cameron D, Brown J, Dent R, Jackisch C, Mackey J, Pivot X, Steger GG, Suter TM, Toi M, Parmar M, Laeufle R, Im YH, et al. Adjuvant bevacizumab-containing therapy in triple-negative breast cancer (BEATRICE): primary results of a randomised, phase 3 trial. The lancet oncology 2013;14: 933-42.

9. Slamon DJ, Swain SM, Buyse M, Martin M, Geyer CE, Im YH, Pienkowski T, Kim SB, Robert NJ, Steger G, Crown J, Verma S, et al. Primary results from $B E T H$, a phase 3 controlled study of adjuvant chemotherapy and trastuzumab \pm bevacizumab in patients with HER2-positive, node-positive or 
high risk node-negative breast cancer. 35TH ANNUAL SAN ANTONIO BREAST CANCER SYMPOSIUM 2013;Abstract nr S1-03.

10. Burstein HJ, Chen YH, Parker LM, Savoie J, Younger J, Kuter I, Ryan PD, Garber JE, Chen H, Campos SM, Shulman LN, Harris LN, et al. VEGF as a marker for outcome among advanced breast cancer patients receiving anti-VEGF therapy with bevacizumab and vinorelbine chemotherapy. Clinical cancer research : an official journal of the American Association for Cancer Research 2008;14: 7871-7.

11. Miles DW, de Haas SL, Dirix LY, Romieu G, Chan A, Pivot X, Tomczak P, Provencher L, Cortes J, Delmar PR, Scherer SJ. Biomarker results from the AVADO phase 3 trial of first-line bevacizumab plus docetaxel for HER2-negative metastatic breast cancer. British journal of cancer 2013;108: 1052-60.

12. Gianni L, Romieu GH, Lichinitser M, Serrano SV, Mansutti M, Pivot X, Mariani P, Andre F, Chan A, Lipatov O, Chan S, Wardley A, et al. AVEREL: a randomized phase III Trial evaluating bevacizumab in combination with docetaxel and trastuzumab as first-line therapy for HER2-positive locally recurrent/metastatic breast cancer. Journal of clinical oncology : official journal of the American Society of Clinical Oncology 2013;31: 1719-25.

13. Schneider BP, Gray RJ, Radovich M, Shen F, Vance G, Li L, Jiang G, Miller KD, Gralow JR, Dickler MN, Cobleigh MA, Perez EA, et al. Prognostic and predictive value of tumor vascular endothelial growth factor gene amplification in metastatic breast cancer treated with paclitaxel with and without bevacizumab; results from ECOG 2100 trial. Clinical cancer research : an official journal of the American Association for Cancer Research 2013;19: 1281-9.

14. Gerger A, El-Khoueiry A, Zhang W, Yang D, Singh H, Bohanes $P$, Ning Y, Winder T, Labonte MJ, Wilson PM, Benhaim L, Paez D, et al. Pharmacogenetic angiogenesis profiling for first-line Bevacizumab plus oxaliplatin-based chemotherapy in patients with metastatic colorectal cancer. Clinical cancer research : an official journal of the American Association for Cancer Research 2011;17: 5783-92.

15. Hansen TF, Christensen R, Andersen RF, Garm Spindler KL, Johnsson $A$, Jakobsen $A$. The predictive value of single nucleotide polymorphisms in the VEGF system to the efficacy of first-line treatment with bevacizumab plus chemotherapy in patients with metastatic colorectal cancer: results from the Nordic ACT trial. International journal of colorectal disease 2012;27: 715-20.

16. Lambrechts D, Claes B, Delmar P, Reumers J, Mazzone M, Yesilyurt BT, Devlieger R, Verslype C, Tejpar S, Wildiers H, de Haas S, Carmeliet $P$, et al. VEGF pathway genetic variants as biomarkers of treatment outcome with bevacizumab: an analysis of data from the AViTA and AVOREN randomised trials. The lancet oncology 2012;13: 724-33.

17. Loupakis F, Cremolini C, Fioravanti A, Orlandi P, Salvatore L, Masi G, Di Desidero T, Canu B, Schirripa M, Frumento P, Di Paolo A, Danesi R, et al. Pharmacodynamic and pharmacogenetic angiogenesis-related markers of first-line FOLFOXIRI plus bevacizumab schedule in metastatic colorectal cancer. British journal of cancer 2011;104: 1262-9.

18. Loupakis F, Cremolini C, Yang D, Salvatore L, Zhang W, Wakatsuki T, Bohanes P, Schirripa M, Benhaim L, Lonardi S, Antoniotti C, Aprile G, et al. 
Prospective validation of candidate SNPs of VEGF/VEGFR pathway in metastatic colorectal cancer patients treated with first-line FOLFIRI plus bevacizumab. PloS one 2013;8: e66774.

19. Schneider BP, Wang M, Radovich M, Sledge GW, Badve S, Thor A, Flockhart DA, Hancock B, Davidson N, Gralow J, Dickler M, Perez EA, et al. Association of vascular endothelial growth factor and vascular endothelial growth factor receptor-2 genetic polymorphisms with outcome in a trial of paclitaxel compared with paclitaxel plus bevacizumab in advanced breast cancer: ECOG 2100. Journal of clinical oncology : official journal of the American Society of Clinical Oncology 2008;26: 4672-8.

20. de Haas S, Delmar P, Bansal AT, Moisse M, Miles DW, Leighl N, Escudier B, Van Cutsem E, Carmeliet P, Scherer SJ, Pallaud C, Lambrechts $D$. Genetic variability of VEGF pathway genes in six randomized phase III trials assessing the addition of bevacizumab to standard therapy. Angiogenesis 2014.

21. Untch M, Loibl S, Bischoff J, Eidtmann H, Kaufmann M, Blohmer JU, Hilfrich J, Strumberg D, Fasching PA, Kreienberg R, Tesch H, Hanusch C, et al. Lapatinib versus trastuzumab in combination with neoadjuvant anthracycline-taxane-based chemotherapy (GeparQuinto, GBG 44): a randomised phase 3 trial. The lancet oncology 2012;13: 135-44.

22. von Minckwitz G, Eidtmann H, Loibl S, Blohmer JU, Costa SD, Fasching PA, Kreienberg R, Hilfrich J, Gerber B, Hanusch C, Fehm T, Strumberg $D$, et al. Integrating bevacizumab, everolimus, and lapatinib into current neoadjuvant chemotherapy regimen for primary breast cancer. Safety results of the GeparQuinto trial. Annals of oncology : official journal of the European Society for Medical Oncology / ESMO 2011;22: 301-6.

23. Huober J, Fasching PA, Hanusch C, Rezai M, Eidtmann H, Kittel K, Hilfrich J, Schwedler K, Blohmer JU, Tesch H, Gerber B, Hoss C, et al. Neoadjuvant chemotherapy with paclitaxel and everolimus in breast cancer patients with non-responsive tumours to epirubicin/cyclophosphamide (EC) +/bevacizumab - results of the randomised GeparQuinto study (GBG 44). European journal of cancer 2013;49: 2284-93.

24. Barrett JC. Haploview: Visualization and analysis of SNP genotype data. Cold Spring Harbor protocols 2009;2009: pdb ip71.

25. Pettersson FH, Anderson CA, Clarke GM, Barrett JC, Cardon LR, Morris AP, Zondervan KT. Marker selection for genetic case-control association studies. Nature protocols 2009;4: 743-52.

26. Salmen J, Neugebauer J, Fasching PA, Haeberle L, Huober J, Wockel A, Rauh C, Schuetz F, Weissenbacher T, Kost B, Stickeler E, Klar M, et al. Pooled analysis of the prognostic relevance of progesterone receptor status in five German cohort studies. Breast cancer research and treatment 2014;148: 143-51.

27. Hegde PS, Jubb AM, Chen D, Li NF, Meng YG, Bernaards C, Elliott $\mathrm{R}$, Scherer SJ, Chen DS. Predictive impact of circulating vascular endothelial growth factor in four phase III trials evaluating bevacizumab. Clinical cancer research : an official journal of the American Association for Cancer Research 2013;19: 929-37.

28. Pentheroudakis G, Kotoula V, Kouvatseas G, Charalambous E, Dionysopoulos D, Zagouri F, Koutras A, Papazisis K, Pectasides D, Samantas E, Dimopoulos MA, Papandreou CN, et al. Association of VEGF-A 
Splice Variant mRNA Expression With Outcome in Bevacizumab-Treated Patients With Metastatic Breast Cancer. Clinical breast cancer 2014.

29. Miles D. MERiDiAN: A phase III, randomized, double-blind study of efficacy, safety, and associated biomarker of bevacizumab plus paclitaxel compared with paclitaxel plus placebo, as first-line therapy of patients with HER2-negative breast cancer [abstract]. Journal of clinical oncology : official journal of the American Society of Clinical Oncology 2013;31: TPS1142.

30. Brogan IJ, Khan N, Isaac K, Hutchinson JA, Pravica V, Hutchinson IV. Novel polymorphisms in the promoter and 5' UTR regions of the human vascular endothelial growth factor gene. Human Immunology 1999;60: 12459.

31. Shahbazi M, Fryer AA, Pravica V, Brogan IJ, Ramsay HM, Hutchinson IV, Harden PN. Vascular endothelial growth factor gene polymorphisms are associated with acute renal allograft rejection. Journal of the American Society of Nephrology : JASN 2002;13: 260-4.

32. Lambrechts D, Storkebaum E, Morimoto M, Del-Favero J, Desmet F, Marklund SL, Wyns S, Thijs V, Andersson J, van Marion I, Al-Chalabi A, Bornes $S$, et al. VEGF is a modifier of amyotrophic lateral sclerosis in mice and humans and protects motoneurons against ischemic death. Nature genetics 2003;34: 383-94.

33. Yang T-P, Beazley C, Montgomery SB, Dimas AS, GutierrezArcelus M, Stranger BE, Deloukas P, Dermitzakis ET. Genevar: a database and Java application for the analysis and visualization of SNP-gene associations in eQTL studies. Bioinformatics 2010;26: 2474-6.

34. Heist RS, Duda DG, Sahani DV, Ancukiewicz M, Fidias P, Sequist LV, Temel JS, Shaw AT, Pennell NA, Neal JW, Gandhi L, Lynch TJ, et al. Improved tumor vascularization after anti-VEGF therapy with carboplatin and nab-paclitaxel associates with survival in lung cancer. Proceedings of the National Academy of Sciences of the United States of America 2015;112: 1547-52.

35. Zhu AX, Ancukiewicz M, Supko JG, Sahani DV, Blaszkowsky LS, Meyerhardt JA, Abrams TA, McCleary NJ, Bhargava P, Muzikansky A, Sheehan S, Regan E, et al. Efficacy, safety, pharmacokinetics, and biomarkers of cediranib monotherapy in advanced hepatocellular carcinoma: a phase II study. Clinical cancer research : an official journal of the American Association for Cancer Research 2013;19: 1557-66.

36. Mina LA, Yu M, Johnson C, Burkhardt C, Miller KD, Zon R. A phase II study of combined VEGF inhibitor (bevacizumab+sorafenib) in patients with metastatic breast cancer: Hoosier Oncology Group Study BRE06-109. Investigational new drugs 2013;31: 1307-10.

37. Mayer EL, Dhakil S, Patel T, Sundaram S, Fabian C, Kozloff M, Qamar R, Volterra F, Parmar H, Samant M, Burstein HJ. SABRE-B: an evaluation of paclitaxel and bevacizumab with or without sunitinib as first-line treatment of metastatic breast cancer. Annals of oncology : official journal of the European Society for Medical Oncology / ESMO 2010;21: 2370-6.

38. Roda JM, Wang Y, Sumner LA, Phillips GS, Marsh CB, Eubank TD. Stabilization of HIF-2alpha induces sVEGFR-1 production from tumorassociated macrophages and decreases tumor growth in a murine melanoma model. Journal of immunology (Baltimore, Md : 1950) 2012;189: 3168-77. 
39. Pruim RJ, Welch RP, Sanna S, Teslovich TM, Chines PS, Gliedt TP, Boehnke M, Abecasis GR, Willer CJ. LocusZoom: regional visualization of genome-wide association scan results. Bioinformatics 2010;26: 2336-7. 
Table 1. Patient characteristics and distribution of $\mathrm{pCR}$

\begin{tabular}{lccc}
\hline & No pCR & & pCR \\
\cline { 2 - 2 } Characteristic & Mean (SD) or & & Mean (SD) or \\
count (\%) & & count (\%) \\
\hline Age (year) & $49.9(10.2)$ & & $47.0(10.1)$ \\
BMl (kg/m $\left.{ }^{2}\right)$ & $25.8(4.8)$ & & $25.9(4.7)$ \\
Treatment arm & & \\
\multicolumn{1}{c}{ EC-T } & $606(83.7)$ & & $118(16.3)$ \\
ECB-TBEV & $586(80.4)$ & & $143(19.6)$
\end{tabular}

Lymph-node status

$\begin{array}{lll}\mathrm{cN}+ & 710(83.6) & 139(16.4) \\ \mathrm{cNO} & 482(79.8) & 122(20.2)\end{array}$

Histology

$\begin{array}{lrr}\text { Ductal } & 944(81.2) & 219(18.8) \\ \text { Lobular } & 137(94.5) & 8(5.5) \\ \text { Other } & 111(76.6) & 34(23.4)\end{array}$

Hormone receptor status

$\begin{array}{lrr}\text { Negative } & 318(64.0) & 179(36.0) \\ \text { Positive } & 874(91.4) & 82(8.6)\end{array}$

Tumor stage

$\begin{array}{lrr}\text { cT1 } & 174(74.4) & 60(25.6) \\ \text { cT2 } & 800(81.5) & 182(18.5) \\ \text { cT3 } & 76(91.6) & 7(8.4) \\ \text { cT4 } & 142(92.2) & 12(7.8)\end{array}$

Grading

$\begin{array}{rrr}\text { G1 } & 50(94.3) & 3(5.7) \\ \text { G2 } & 691(89.6) & 80(10.4) \\ \text { G3 } & 451(71.7) & 178(28.3)\end{array}$

BEV, bevacizumab; BMI, body mass index; EC, epirubicin and cyclophosphamide; pCR, pathological complete remission; SD, standard deviation; $\mathrm{T}$, paclitaxel. 
Table 2. Ten SNPs with the lowest $P$ values for treatment by genotype interaction relative to pathological complete response. Odds ratios for bevacizumab versus standard treatment by genotype are shown

\begin{tabular}{|c|c|c|c|c|c|c|c|c|c|}
\hline \multirow[b]{2}{*}{ SNP } & \multirow[b]{2}{*}{$\begin{array}{l}\text { Major allele/ } \\
\text { minor allele }\end{array}$} & \multirow[b]{2}{*}{ Gene } & \multirow[b]{2}{*}{ Chr } & \multirow[b]{2}{*}{ Position } & \multirow[b]{2}{*}{ MAF } & \multirow[b]{2}{*}{$P$ value $^{1}$} & \multicolumn{3}{|c|}{ Odds ratio ( $95 \%$ confidence intervals) } \\
\hline & & & & & & & $\begin{array}{c}\text { Homozygous } \\
\text { common }\end{array}$ & Heterozygous & $\begin{array}{c}\text { Homozygous } \\
\text { rare }\end{array}$ \\
\hline rs833058 & $\mathrm{G} / \mathrm{A}$ & VEGF-A & 6 & 43764117 & 0.39 & 0.003 & $2.36(1.49,3.75)$ & $1.21(0.89,1.65)$ & $0.62(0.34,1.13)$ \\
\hline rs699947 & $\mathrm{A} / \mathrm{C}$ & VEGF-A & 6 & 43768652 & 0.50 & 0.032 & $2.24(1.31,3.84)$ & $1.40(1.04,1.88)$ & $0.87(0.53,1.45)$ \\
\hline rs3025039 & $\mathrm{C} / \mathrm{T}$ & VEGF-A & 6 & 43784799 & 0.17 & 0.040 & $1.71(1.20,2.44)$ & $0.97(0.62,1.53)$ & $0.55(0.22,1.41)$ \\
\hline rs3025030 & $\mathrm{G} / \mathrm{C}$ & VEGF-A & 6 & 43782850 & 0.16 & 0.041 & $1.72(1.20,2.45)$ & $0.98(0.62,1.54)$ & $0.56(0.22,1.42)$ \\
\hline rs7995976 & $C / A$ & FLT1 & 13 & 28366923 & 0.25 & 0.049 & $1.06(0.72,1.56)$ & $1.73(1.18,2.53)$ & $2.82(1.28,6.19)$ \\
\hline rs4953344 & $\mathrm{T} / \mathrm{C}$ & EPAS1 & 2 & 46325319 & 0.18 & 0.058 & $1.65(1.15,2.35)$ & $0.95(0.59,1.54)$ & $0.55(0.21,1.48)$ \\
\hline rs3768728 & $\mathrm{T} / \mathrm{C}$ & EPAS1 & 2 & 46363652 & 0.15 & 0.067 & $1.61(1.14,2.27)$ & $0.90(0.52,1.56)$ & $0.50(0.16,1.54)$ \\
\hline rs1374749 & $\mathrm{G} / \mathrm{A}$ & EPAS1 & 2 & 46369294 & 0.46 & 0.073 & $1.88(1.16,3.03)$ & $1.28(0.94,1.72)$ & $0.87(0.50,1.51)$ \\
\hline rs3025033 & $A / G$ & $V E G F-A$ & 6 & 43783338 & 0.18 & 0.074 & $1.68(1.17,2.41)$ & $1.05(0.68,1.61)$ & $0.65(0.27,1.58)$ \\
\hline rs1629140 & $\mathrm{G} / \mathrm{A}$ & EGLN3 & 14 & 33944620 & 0.42 & 0.103 & $1.84(1.16,2.93)$ & $1.30(0.96,1.76)$ & $0.92(0.52,1.61)$ \\
\hline
\end{tabular}

${ }^{1}$ Uncorrected $P$ value for treatment by genotype interaction based on multiple logistic regression model with the additional predictors age, body mass index, tumor stage, lymph-node status, histology, grading, and hormone receptor status. None of the Bonferroni-Holm corrected $P$ values were significant: $P=0.34$ for rs 833058 and $P=1.00$ for all other SNPs.

Chr, chromosome; MAF, minor allele frequency; SNP, single nucleotide polymorphism. 
Table 3. Hormone receptor-specific associations between treatment, genotype, and pathological complete response. Odds ratios for bevacizumab versus standard treatment by genotype are shown

\begin{tabular}{|c|c|c|c|c|}
\hline \multirow[b]{2}{*}{ SNP $^{1}$} & \multirow[b]{2}{*}{ HR } & \multicolumn{3}{|c|}{ Odds ratio (95\% confidence intervals) } \\
\hline & & $\begin{array}{c}\text { Homozygous } \\
\text { common }\end{array}$ & Heterozygous & $\begin{array}{c}\text { Homozygous } \\
\text { rare }\end{array}$ \\
\hline rs3025039 & $\mathrm{HR}-$ & $2.17(1.38,3.41)$ & $1.04(0.56,1.93)$ & $0.50(0.14,1.77)$ \\
\hline (VEGFA) & $\mathrm{HR}+$ & $1.21(0.67,2.16)$ & $0.89(0.47,1.68)$ & $0.66(0.18,2.40)$ \\
\hline rs3025030 & HR- & $2.24(1.42,3.53)$ & $1.07(0.58,1.99)$ & $0.52(0.15,1.82)$ \\
\hline (VEGFA) & $\mathrm{HR}+$ & $1.16(0.65,2.07)$ & $0.86(0.46,1.63)$ & $0.64(0.18,2.33)$ \\
\hline rs3768728 & $\mathrm{HR}-$ & $2.36(1.50,3.70)$ & $0.81(0.39,1.68)$ & $0.28(0.06,1.23)$ \\
\hline (EPAS1) & $\mathrm{HR}+$ & $0.91(0.53,1.57)$ & $1.06(0.46,2.44)$ & $1.22(0.22,6.86)$ \\
\hline rs1374749 & $\mathrm{HR}-$ & $3.20(1.68,6.07)$ & $1.55(1.05,2.28)$ & $0.75(0.37,1.52)$ \\
\hline (EPAS1) & $\mathrm{HR}+$ & $0.91(0.45,1.87)$ & $1.00(0.62,1.61)$ & $1.09(0.44,2.71)$ \\
\hline rs3025033 & $\mathrm{HR}-$ & $2.21(1.39,3.51)$ & $1.14(0.63,2.04)$ & $0.59(0.18,1.96)$ \\
\hline (VEGFA) & $\mathrm{HR+}$ & $1.15(0.64,2.09)$ & $0.90(0.49,1.63)$ & $0.70(0.21,2.34)$ \\
\hline
\end{tabular}

\footnotetext{
${ }^{1}$ Subset of SNPs from Table 2. Only those SNPs were included for which the logistic regression model with hormone receptor interaction terms a had better (i.e., smaller) Akaike information criterion than the logistic regression model without hormone receptor interaction terms.
}

Abbreviations: HR, hormone receptor; SNP, single nucleotide polymorphism. 
Figure 1. The VEGFA gene with - $\log$ of $P$ values for genes in the area of VEGFA (figure generated with LocusZoom ${ }^{39}$ )

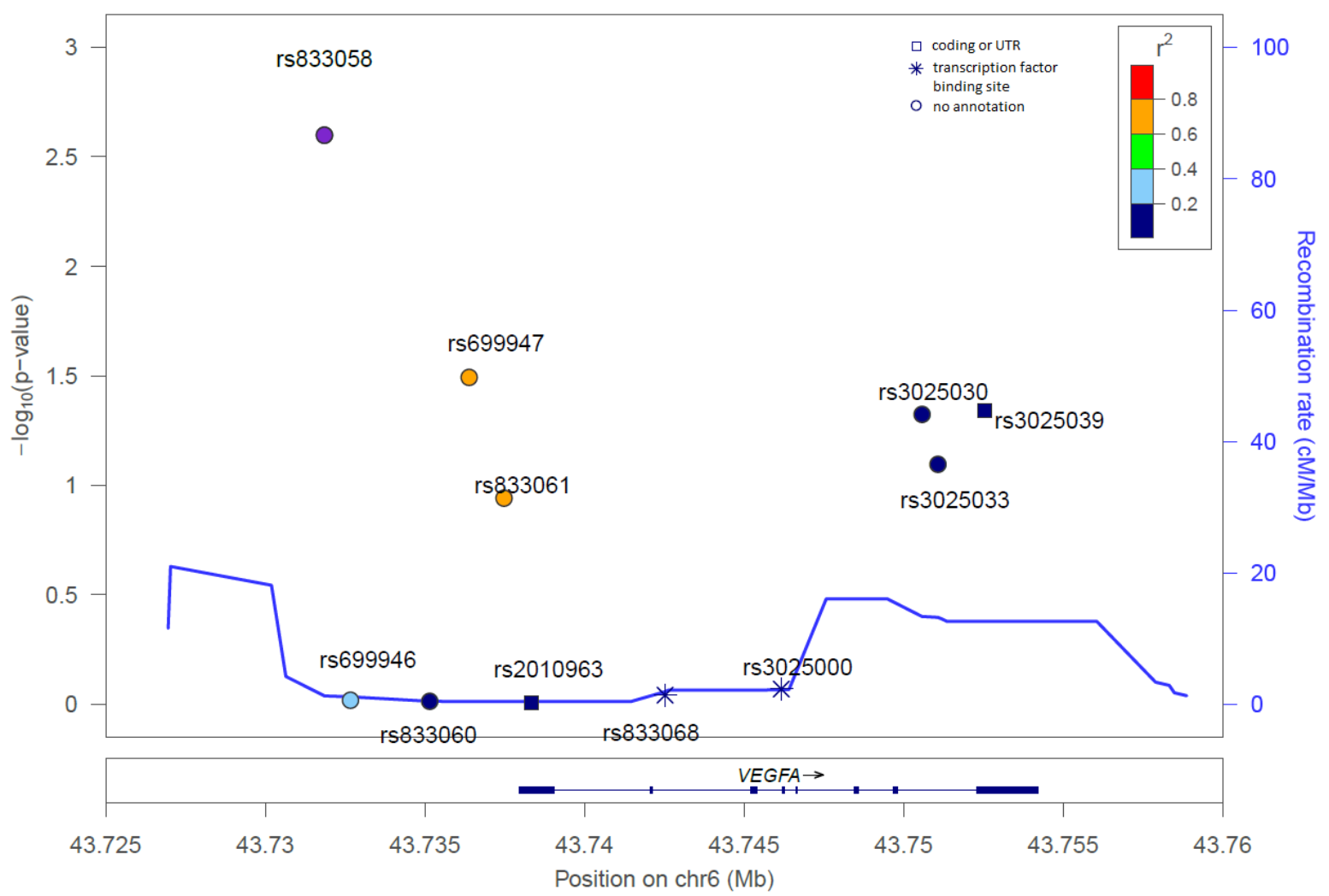

